\title{
Population-based utilization and costs associated with tyrosine kinase inhibitors for first-line treatment of chronic myelogenous leukemia among elderly patients
}

Donna R Rivera, PharmD, MSc; Lindsey Enewold, PhD, MPH; Michael J Barrett, MS; Matthew P Banegas, PhD, MPH; Kelly K Filipski, PhD, MPH; Andrew N Freedman, PhD; Clara K Lam, PhD, MPH; and Angela Mariotto, PhD

\section{What is already known about this subject}

- Multiple tyrosine kinase inhibitor (TKI) therapeutic options are approved for the treatment of chronic myelogenous leukemia (CML); however, there are few population-based studies assessing treatment utilization patterns.

- Early TKI initiation is linked to improved drug response and survival.

- Amid rising health care costs, the cost of guideline-recommended targeted treatments such as TKIs is significant for patients and the health care system.

\section{ABSTRACT}

BACKGROUND: Following approval of imatinib, a breakthrough tyrosine kinase inhibitor (TKI), survival significantly improved by more than $20 \%$ since 2001 among treated chronic

\author{
What this study adds \\ - This study provides an updated real- \\ world examination of contemporary TKI \\ (imatinib, dasatinib, nilotinib) utilization \\ patterns within 1 year of diagnosis \\ among elderly (aged 65+ years) CML \\ patients diagnosed between 2007-2015 \\ on traditional Medicare (fee for service) \\ and Medicare Advantage (health \\ maintenance organizations). \\ - Study findings show an increase in TKI \\ use from $66 \%$ in 2007 to $79 \%$ in 2015 , \\ along with an increased diffusion of \\ newer agents dasatinib and nilotinib; \\ $60 \%$ of included CML patients initiated \\ TKI treatment within 3 months of \\ diagnosis. \\ - This study shows continued disparate \\ use of TKIs-initiation was lower in CML \\ patients aged 75 years and older and \\ those who were low-income subsidy \\ (LIS) ineligible through Medicare Part \\ D; patients who are LIS ineligible face \\ considerable out-of-pocket costs to \\ obtain this necessary treatment.
}

\author{
Author affiliations \\ Donna R Rivera, PharmD, MSc; Clara K \\ Lam, PhD, MPH; and Angela Mariotto, \\ PhD, National Cancer Institute, Division of \\ Cancer Control and Population Sciences, \\ Surveillance Research Program, Rockville, \\ MD. Lindsey Enewold, PhD, MPH, National \\ Cancer Institute, Division of Cancer Control \\ and Population Sciences, Health Care \\ Delivery Research Program, Rockville, MD; \\ Michael J Barrett, MS, Information \\ Management Services, Calverton, MD; and \\ Matthew P Banegas, PhD, MPH, Kaiser \\ Permanente Center for Health Research, \\ Portland, OR. Kelly K Filipski, PhD, MPH, \\ and Andrew N Freedman, PhD, National \\ Cancer Institute, Division of Cancer Control \\ and Population Sciences, Epidemiology and \\ Genomics Research Program, Rockville, MD. \\ AUTHOR CORRESPONDENCE: \\ Donna R Rivera, 240.276.6740, \\ donna.rivera@fda.hhs.gov
}

J Manag Care Spec Pharm. 2020;26(12):1494-504

Copyright $\odot 2020$, Academy of Managed Care Pharmacy. All rights reserved.

their escalating costs and recommendations for maintenance therapy.

OBJECTIVE: To assess the utilization patterns of first-line TKIs, overall and by specific agent, among elderly CML patients in the United States, and the cost implications. myelogenous leukemia (CML) patients. Subsequently, more expensive second-generation TKIs with varying selectivity profiles have been approved. Population-based stud ies are needed to evaluate the real-world utilization of TKI therapies, particularly given 
METHODS: CML patients aged 65 years and older at diagnosis between 2007 and 2015 were identified from population-based cancer registries in the linked Surveillance, Epidemiology, and End Results (SEER)-Medicare database. The percentage of CML patients receiving imatinib, dasatinib, or nilotinib within the first year of diagnosis was calculated along with time to first-line treatment initiation. Bivariate comparisons and Cox proportional hazards models were used to identify factors associated with TKI initiation. Average monthly patient responsibility, including patient out-of-pocket (OOP) costs, stratified by Part D low-income subsidy (LIS) status were also calculated.

RESULTS: Among the 1,589 CML patients included, receipt of any TKI within 1 year of diagnosis increased from $66.2 \%$ to $78.9 \%$. In 2015 , the distribution of first-line TKI therapies was $41.3 \%$ imatinib, $28.3 \%$ dasatinib, and $9.3 \%$ nilotinib. Almost $60 \%$ of patients initiated TKI treatment within 3 months of diagnosis. Multivariable analysis indicated that TKI use in the first year was lower among the very elderly (aged $>75$ years vs. $65-69$ years: $\mathrm{HR}=0.72 ; 95 \% \mathrm{Cl}=0.63-0.83$ ), patients with more comorbidities (Hierarchical Condition Category risk score $>2$ vs. $\mathrm{HR}=0.74,95 \% \mathrm{Cl}=0.62-0.88)$, and patients ineligible for $\mathrm{LIS}(\mathrm{HR}=0.75$; $95 \% \mathrm{Cl}=0.65-0.87$ ). Average monthly patient OOP cost was significantly lower for LIS-eligible versus LIS-ineligible patients: imatinib (2016: \$12 vs. \$487), dasatinib (2016: \$34 vs. \$557), and nilotinib (2016: \$1 vs. \$526).

CONCLUSIONS: TKI use has increased significantly since 2007. While imatinib remained the most frequently prescribed first-line agent, by 2015 newer TKIs represented one third of the market share. Utilization patterns indicated persistent age, comorbidity, and financial barriers. TKI use is indicated for long-term therapy, and increased adoption of newer, more expensive agents raises concerns about the sustained affordability of CML treatment, particularly among unsubsidized patients.

Chronic myelogenous leukemia (CML) is a hematological malignancy that has had a relatively stable incidence rate over the past several decades. ${ }^{1}$ In 2019, an estimated 8,990 new CML cases were diagnosed in the United States, representing about $15 \%$ of leukemia diagnoses and $0.5 \%$ of all cancer diagnoses. ${ }^{2} \mathrm{CML}$ primarily affects older adults-median age at diagnosis is 64 years and mortality is highest among patients aged $75-84$ years. ${ }^{1}$

The 5-year relative survival rate for CML cases diagnosed in 2007-2013 was 68.0\%, which is a vast improvement from the $47.3 \%$ rate among cases diagnosed in 1999-2000, and even more so than those diagnosed in 1975-1977 (21.8\%). ${ }^{1}$ The statistically significant improvement in survival is largely attributed to the introduction of new molecularly targeted therapies, specifically tyrosine kinase inhibitors (TKIs). ${ }^{3,4}$ CML is categorized by the presence of a Philadelphia chromosome $(\mathrm{Ph}+)$; more than $95 \%$ of $\mathrm{CML}$ cases have this reciprocal translocation of chromosomes 9 and 22 that produces the aberrant BCR-ABL tyrosine kinase signaling protein.

In 2001, Gleevec (imatinib mesylate) became the first oral therapeutic approved for the treatment of CML. ${ }^{5}$ Imatinib is a small molecule TKI that inhibits the BCR-ABL tyrosine kinase (and additional kinase domains) in $\mathrm{Ph}+\mathrm{CML}$. Since the introduction of imatinib, the role of molecularly targeted therapy in CML has increased with the development of second-generation TKIs, such as Sprycel (dasatinib, 2006), Tasigna (nilotinib, 2007), Iclusig (ponatinib, 2012), and Bosulif (bosutinib, 2012). The second-generation TKIs have increased selectivity, potency, and variant kinase inhibition, but they are more expensive.

The cost differential between imatinib and the secondgeneration TKIs may be more pronounced in recent years because a generic version of imatinib became available in 2016. Nonetheless, the additional therapeutic choices have been especially important for patients who develop resistance to imatinib. ${ }^{6}$ National Comprehensive Cancer Network guidelines v1.2019 recommend the use of imatinib, dasatinib, nilotinib, or bosutinib as first-line treatment options across all 3 clinical phases (chronic, accelerated, and blast) of CML.?

To date, there have been a handful of population-based studies among patients aged 65 years and older at diagnosis that have assessed the use of TKIs for the treatment of CML. ${ }^{8-11}$ TKI initiation was found to be suboptimal $(<70 \%)$, especially among older patients, patients with comorbidities, and patients without Medicare Part D low-income subsidies (LIS). ${ }^{8,9}$ TKI adherence was also shown to be lower and even delayed among patients without LIS. , $^{810}$

Although TKI use has been associated with higher costs, TKIs have demonstrated benefit for cost, and adequate TKI drug exposure was associated with increased 5-year survival compared with traditional chemotherapy or no treatment. ${ }^{11}$ Because these previous studies were limited to patient data through 2012, it was unclear if these same costs trends would be observed in more recent years.

This study aimed to assess more recent TKI utilization patterns and the associated health care and patient-level costs. Data through 2016 were included, allowing the ability to assess the initial impact of generic imatinib. Variations in utilization patterns between patients enrolled in traditional Medicare (fee for service) and Medicare Advantage (e.g., health maintenance organizations) and by LIS status were also assessed. 


\section{Methods}

\section{DATA SOURCES}

The study used the Surveillance, Epidemiology, and End Results (SEER) cancer registry data linked to Medicare enrollment and claims data (SEER-Medicare). ${ }^{12}$ The SEER data are collected by National Cancer Institute (NCI) funded population-based cancer registries and, at the time of analyses, captured $28 \%$ of the U.S. population. Each registry routinely collects data on all newly diagnosed cancers within their specified geographic area, including patient demographics, cancer site (International Classification of Diseases for Oncology, Third Edition [ICD-O-3]) codes, date of diagnosis, tumor characteristic (e.g., stage or histology), first-course of treatment, prior cancers, and survival.

The Medicare data include information pertaining to monthly enrollment in traditional Medicare (referred to here as fee for service [FFS]) or Medicare Advantage (referred to here as health maintenance organizations [HMOs]) and type of coverage (Part A: inpatient, Part B: outpatient, and Part D: prescription medication).

Administrative claims are available for prescription medications for all Medicare beneficiaries (FFS and HMO) enrolled in Medicare Prescription Drug plans (Part D), and for inpatient and outpatient services provided to FFS beneficiaries enrolled in Parts A and B, respectively.

Although Medicare beneficiaries have been able to enroll in Part D since 2006, Part D claims are available in SEER-Medicare starting in 2007. The Part D data were used to identify first-line TKI use and determine whether each beneficiary was eligible for an LIS to defray out-of-pocket (OOP) costs..$^{12}$ The most recent linkage of SEER-Medicare data included Part D claims from 2007-2016 and cancer cases diagnosed through 2015.

\section{PATIENT SELECTION}

This study included patients aged 65 years and older with a first primary cancer diagnosis of CML (ICD-O-3 codes 9863 and 9875-9876) between 2007 and 2015. ${ }^{13}$ Patients were excluded if there were any claims for a TKI more than 2 months before their listed CML diagnosis date; in these instances, there were concerns that the registry diagnosis date (available only as month and year) was inaccurate and could result in miscalculation of time to TKI initiation and/or that the CML diagnosis was not the first cancer diagnosis. Patients were required to have continuous enrollment in Medicare Parts A, B, and D from the time of diagnosis through death or end of follow-up (12 months after diagnosis), whichever occurred first.

\section{PATIENT FACTORS}

The analysis included assessment of selected patient characteristics that may be associated with receipt of a TKI: age at diagnosis, sex, race, HMO enrollment at diagnosis, and LIS eligibility at diagnosis. Additionally, previous health status, which can influence treatment decisions, was assessed for each patient based on the Centers for Medicare \& Medicaid Services Hierarchical Condition Category (CMS-HCC) risk scores. ${ }^{14,15}$ Briefly, the CMS-HCC risk scores are calculated annually for all Medicare beneficiaries, regardless of HMO enrollment, based on a linear regression model that includes age, sex, and the occurrence of specific diagnoses in the previous year. CMS uses these risk scores to adjust payments made to HMO plans. Plans that include more beneficiaries with poor health status (high risk) are reimbursed at a higher level than plans that include more beneficiaries with good health status (low risk). The CMS-HCC risk score has been shown to outperform other commonly used comorbidity indices (e.g., Charlson and Elixhauser) for predicting in-hospital and 6-month mortality. ${ }^{16}$ In this study, CMS-HCC risk scores were divided into $<1,1-2$, and $>2$ categories, with a higher score indicating a poorer health status (higher risk).

Information on phase or severity of CML was not available, since the registries do not collect cytogenetic information, and claims data were not uniformly available for all eligible study participants. Diagnosis codes are not included on Part D claims, and although diagnosis codes are included on Parts A and B claims, these claims were only available for eligible FFS participants.

\section{TKI ASSESSMENT}

All TKIs that were approved by the U.S. Food and Drug Administration (FDA) through 2016 for the treatment of CML (imatinib, dasatinib, nilotinib, ponatinib, and bosutinib) were identified using the National Drug Code numbers reported on the Part D claims (Supplementary Table 1, available in online article). The analysis focused on the first TKI use within 12 months of diagnosis. The number of patients who received first-line ponatinib or bosutinib, both approved in 2012, was very small $(n<11)$; therefore, use of these agents was not considered, and these patient were excluded from the analysis. Although patients could have received more than 1 TKI agent during the study period, patients were assigned to the first TKI agent received. Patients with no recorded TKI claims within 12 months of diagnosis were categorized as "none" in the analysis.

\section{ANALYTIC APPROACH}

The number and percentage of patients receiving first-line TKI therapy within 12 months of diagnosis overall, and by 


\section{TABLE 1}

Characteristics of Patients Aged $\geq 65$ Years at Diagnosis with CML by Receipt of First-Line TKI Within the First Year After Diagnosis, 2007-2015 SEER-Medicare

\begin{tabular}{l|c|c|c|c|c|c|c|c|c}
\hline \multirow{2}{*}{} & \multicolumn{8}{|c}{ Tyrosine Kinase Inhibitor } \\
\cline { 2 - 10 } & Total & \multicolumn{2}{|c|}{ Imatinib } & \multicolumn{2}{c}{ Dasatinib } & \multicolumn{2}{c}{ Nilotinib } & \multicolumn{2}{c}{ None } \\
\cline { 2 - 10 } & $\mathbf{N}$ & $\mathbf{N}$ & $\%$ & $\mathbf{N}$ & $\%$ & $\mathbf{N}$ & $\%$ & $\mathbf{N}$ & $\%$ \\
\hline Total & 1,589 & 738 & 46.4 & 237 & 14.9 & 127 & 8.0 & 487 & 30.6 \\
\hline
\end{tabular}

Age, years ${ }^{a}$

\begin{tabular}{l|r|r|r|r|r|r|r|r|r}
\hline $65-69$ & 457 & 226 & 49.5 & 97 & 21.2 & 45 & 9.8 & 89 & 19.5 \\
\hline $70-74$ & 332 & 156 & 47.0 & 62 & 18.7 & 32 & 9.6 & 82 & 24.7 \\
\hline $75+$ & 800 & 356 & 44.5 & 78 & 9.8 & 50 & 6.3 & 316 & 39.5 \\
\hline Sex & 788 & 354 & 44.9 & 120 & 15.2 & 74 & 9.4 & 240 & 30.5 \\
\hline Male & 801 & 384 & 47.9 & 117 & 14.6 & 53 & 6.6 & 247 & 30.8 \\
\hline
\end{tabular}

Race

\begin{tabular}{|l|r|r|r|c|c|c|c|c|c|}
\hline White & 1,315 & 617 & 46.9 & 186 & 14.1 & 105 & 8.0 & 407 & 31.0 \\
\hline Black & 142 & 70 & 49.3 & $c$ & $c$ & $c$ & $c$ & 42 & 29.6 \\
\hline Other & 132 & 51 & 38.6 & $c$ & $c$ & $c$ & $c$ & 38 & 28.8 \\
\hline
\end{tabular}

HMO enrollment ${ }^{a}$

\begin{tabular}{l|l|l|l|r|r|r|r|r|r}
\hline Yes & 619 & 315 & 50.9 & 90 & 14.5 & 41 & 6.6 & 173 & 27.9 \\
\hline No & 970 & 423 & 43.6 & 147 & 15.2 & 86 & 8.9 & 314 & 32.4 \\
\hline
\end{tabular}

LIS eligibility

\begin{tabular}{l|r|r|r|r|r|r|r|r|r}
\hline Yes & 429 & 210 & 49.0 & 58 & 13.5 & 35 & 8.2 & 126 & 29.4 \\
\hline No & 1,160 & 528 & 45.5 & 179 & 15.4 & 92 & 7.9 & 361 & 31.1 \\
\hline
\end{tabular}

Previous health status ${ }^{\text {b }}$

\begin{tabular}{l|r|r|r|r|r|r|r|r|r}
\hline$<1$ (good) & 763 & 361 & 47.3 & 146 & 19.1 & 65 & 8.5 & 191 & 25.0 \\
\hline $1-2$ & 506 & 244 & 48.2 & 60 & 11.9 & 39 & 7.7 & 163 & 32.2 \\
\hline$>2$ (poor) & 319 & 132 & 41.4 & 31 & 9.7 & 23 & 7.2 & 133 & 41.7 \\
\hline
\end{tabular}

${ }^{a}$ At CML diagnosis.

${ }^{b} \mathrm{CMS}$ HCC risk score.

'Cell sizes suppressed because of confidentiality concerns.

$C M L=$ chronic myelogenous leukemia; $C M S H C C=$ Centers for Medicare \& Medicaid Services Hierarchical Condition Categories; $\mathrm{HMO}=$ health maintenance organization; LIS=low-income subsidy;

SEER $=$ Surveillance, Epidemiology, and End Results; TKI = tyrosine kinase inhibitor.

specific agent and patient factors (including age, sex, race, HMO enrollment, LIS eligibility, CMS-HCC risk score, and year of diagnosis) were assessed. For analytic purposes, patients $(n=12)$ who had a first TKI claim that preceded the registry diagnosis date within 2 months were considered to have received a TKI within the month of CML diagnosis. Temporal trends in TKI utilization and time to TKI initiation were based on person-years, which were calculated from CML diagnosis date to end of follow-up, or death, to account for the contributed study time of each patient. A multivariate Cox regression model was used to estimate the hazard ratios (HRs) and 95\% CIs and identify variables that were associated with the TKI initiation rate. The Cox proportional hazards assumption was assessed by comparing Kaplan-Meier survival curves stratified by covariate categories.

Average monthly (30-day) health care TKI costs (e.g., Medicare payments for drug ingredients and dispensing fees) and patient responsibility, including OOP costs associated with receipt of TKI, were calculated based on the Part D claims. If a TKI claim was submitted for a 90-day supply then the listed cost was divided by 3 to calculate the estimated monthly cost. Monthly costs were then annualized to evaluate yearly trends. Monthly costs for 2016 were investigated to assess the impact of generic imatinib. Costs were not adjusted for inflation and are reported in 2007-2016 U.S. dollars.

All statistical analyses were considered statistically significant if $P$ values were less than 0.05 and were conducted using SAS version 9.4 (SAS Institute, Cary, NC).

\section{Results}

The final cohort included 1,589 patients diagnosed with CML between 2007-2015 (Table 1). More than half of the cohort $(n=800)$ was aged 75 years or older at diagnosis; $83 \%$ of patients were White; and there were roughly equivalent numbers of patients by sex. At diagnosis, approximately $39 \%$ were enrolled in an HMO; 27\% were LIS eligible; and $48 \%$ had an HCC score less than 1 (good health status). Imatinib was the most frequently prescribed TKI (46.4\%) over the entire study period, followed by dasatinib (14.9\%) and nilotinib (8.0\%). About one third (30.6\%) of the cohort did not receive a TKI within 12 months of CML diagnosis.

In $2007,66.2 \%$ of patients received a TKI within a year of their CML diagnosis, increasing to $78.9 \%$ in 2015 (Figure 1). Imatinib was the most commonly used first-line agent across all 


\section{FIGURE 1 Use of First TKI Agents Within 1 Year After Diagnosis Among Patients Aged $\geq 65$ Years at Diagnosis with CML, 2007-2015 SEER-Medicare ${ }^{a}$}
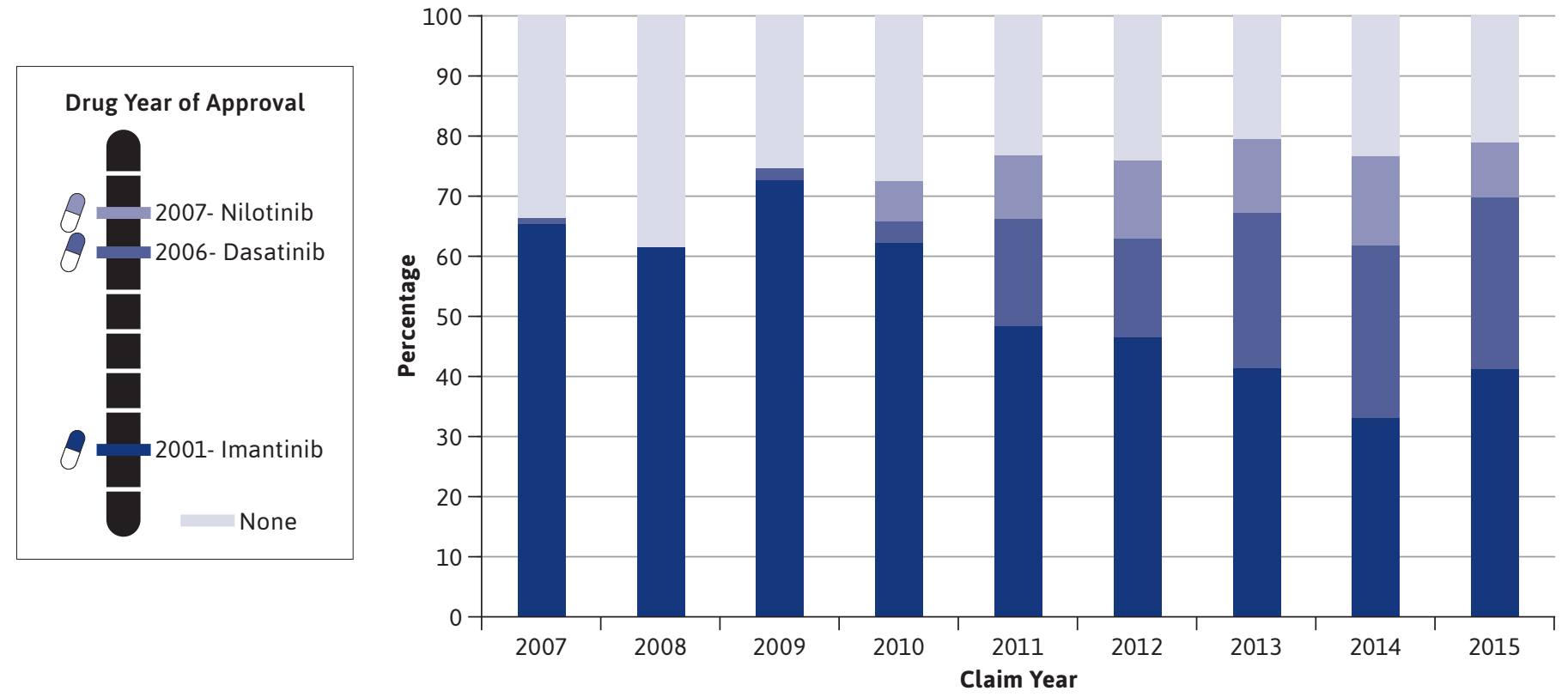

${ }^{a}$ Based on person-years from the time of diagnosis to death or 12 months, whichever occurred first.

$\mathrm{CML}=$ chronic myelogenous leukemia; SEER = Surveillance, Epidemiology, and End Results; TKI=tyrosine kinase inhibitor.

years, with peak usage in 2009. Starting in 2010, there was a marked decline in imatinib use as the adoption of second-generation TKIs began to diffuse into clinical practice (subsequent to FDA approvals). By 2015, imatinib use had decreased to $41.3 \%$, while dasatinib and nilotinib had increased to $28.3 \%$ and $9.3 \%$, respectively. Over the study period, approximately $60 \%$ of CML patients received a TKI within 3 months of diagnosis (Supplementary Figure 1, available in online article), and over $66 \%$ of the patients received a TKI within 12 months of diagnosis.

The multivariate Cox regression model indicated slower TKI initiation within the first year among older patients $>75$ years vs. $65-69$ years: $\mathrm{HR}=0.72,95 \% \mathrm{CI}=0.63-0.83, \mathrm{P}<0.01$; Table 2) and patients who were LIS ineligible (HR $=0.75,95 \%$ $\mathrm{CI}=0.65-0.87), \mathrm{P}<0.01)$. Stratified analysis indicated that the association between TKI initiation and LIS status was not dependent on HMO status (LIS ineligible vs. eligible, FFS: $\mathrm{HR}=0.80,95 \% \mathrm{CI}=0.67-0.95, \mathrm{P}<0.01$; $\mathrm{HMO}: \mathrm{HR}=0.61$, $95 \% \mathrm{CI}=0.47-0.80, \mathrm{P}<0.01$, data not shown). Poorer health status, as assessed by CMS-HCC risk score ( $>2$ vs. 0 ), was also associated with a slower rate of TKI initiation $(\mathrm{HR}=0.74$, 95\% CI $=0.62-0.88, \mathrm{P}<0.01$ ), whereas later diagnosis year was associated with a quicker rate of initiation (2015 vs. 2007: $\mathrm{HR}=1.42,95 \% \mathrm{CI}=1.08-1.88, \mathrm{P}=0.01$ ). The variation in rate of TKI initiation by sex, race, and HMO enrollment at diagnosis was not statistically significant.

Average monthly Medicare payments increased over the study period for each TKI (imatinib 2007: \$2,890, 2016 \$8,421; dasatinib 2009: \$6,106, 2016: \$,8904; and nilotinib 2010: $\$ 7,722,2016$ : $\$ 9,368$; Figure 2). Average monthly OOP costs varied over time, with second-generation TKIs tending to be more expensive than imatinib; these costs became more stable in later years (Figure 2).

Although the difference in average monthly OOP cost by LIS status tended to converge over time, in 2016, mean OOP costs remained substantially lower among LISeligible versus LIS-ineligible patients (imatinib: \$21 vs. \$487, dasatinib: \$34 vs. \$557, and nilotinib: \$1 vs. \$526; Figure 3). The market introduction of the generic imatinib formulation occurred in early 2016; the average monthly Medicare payment for the branded drug ranged from $\$ 9,954$ to $\$ 7,404$ and for generic imatinib from $\$ 8,571$ to $\$ 7,155$ (data not shown). There were fluctuations in the average monthly OOP costs for imatinib after generic introduction, 


\section{TABLE 2}

Factors Associated with Time to First

TKI Among Patients Aged $\geq 65$ Years at Diagnosis with CML, 2007-2015 SEER-Medicare

\begin{tabular}{|c|c|c|c|c|}
\hline & $H \mathbf{R}^{\mathbf{a}}$ & \multicolumn{2}{|c|}{$95 \% \mathrm{Cl}$} & P Value \\
\hline \multicolumn{5}{|l|}{ Age, years ${ }^{b}$} \\
\hline $65-69$ & 1.00 & ref & & \\
\hline $70-74$ & 0.92 & 0.78 & 1.08 & 0.28 \\
\hline$\geq 75$ & 0.72 & 0.63 & 0.83 & $<0.01$ \\
\hline \multicolumn{5}{|l|}{ Sex } \\
\hline Male & 1.00 & ref & & \\
\hline Female & 0.99 & 0.88 & 1.11 & 0.84 \\
\hline \multicolumn{5}{|l|}{ Race } \\
\hline White & 1.00 & ref & & \\
\hline Black & 0.87 & 0.71 & 1.08 & 0.21 \\
\hline Other & 1.00 & 0.80 & 1.24 & 0.99 \\
\hline \multicolumn{5}{|c|}{ HMO enrollment ${ }^{\mathrm{b}}$} \\
\hline Yes & 1.00 & ref & & \\
\hline No & 0.88 & 0.78 & 1.00 & 0.05 \\
\hline \multicolumn{5}{|c|}{ LIS eligibility } \\
\hline Yes & 1.00 & ref & & \\
\hline No & 0.75 & 0.65 & 0.87 & $<0.01$ \\
\hline \multicolumn{5}{|c|}{ Previous health status $^{c}$} \\
\hline$<1$ (good) & 1.00 & ref & & \\
\hline 1 to 2 & 0.93 & 0.81 & 1.07 & 0.29 \\
\hline$>2$ (poor) & 0.74 & 0.62 & 0.88 & $<0.01$ \\
\hline \multicolumn{5}{|c|}{ Year of diagnosis } \\
\hline 2007 & 1.00 & ref & & \\
\hline 2008 & 0.86 & 0.63 & 1.18 & 0.36 \\
\hline 2009 & 1.15 & 0.84 & 1.57 & 0.39 \\
\hline 2010 & 1.16 & 0.86 & 1.57 & 0.34 \\
\hline 2011 & 1.27 & 0.95 & 1.70 & 0.11 \\
\hline 2012 & 1.18 & 0.89 & 1.58 & 0.26 \\
\hline 2013 & 1.32 & 1.00 & 1.73 & 0.05 \\
\hline 2014 & 1.27 & 0.96 & 1.68 & 0.09 \\
\hline 2015 & 1.42 & 1.08 & 1.88 & 0.01 \\
\hline
\end{tabular}

aMultivariate Cox regression model that included all listed variables. ${ }^{\mathrm{b} A t}$ diagnosis.

'CMS HCC risk score.

$C M L=$ chronic myelogenous leukemia; $C M S H C C=$ Centers for Medicare \& Medicaid Services Hierarchical Condition Categories; $\mathrm{HMO}=$ health maintenance organization; LIS = low-income subsidy; ref=reference; SEER =Surveillance, Epidemiology, and End Results; TKI = tyrosine kinase inhibitor. with persisting differences between LIS-eligible and LISineligible patients (Figure 3).

\section{Discussion}

The introduction of TKIs has resulted in improved CML survival, especially for elderly patients. ${ }^{3}$ This is especially promising, since elderly patients may not be candidates for traditional high-dose chemotherapy or allogeneic stem cell transplantation, which was the previous standard of care before the introduction of TKIs. Oral TKIs have also allowed patients to have potentially increased quality of life compared with traditional chemotherapy due to greater autonomy, use of a preferred modality of care, and side effect reduction. ${ }^{17-22}$

Our analysis found that there has been a marked increase in use of TKIs in patients aged over 65 years, as evidenced by $79 \%$ of included CML patients initiating TKI therapy within 1 year of diagnosis in 2015. However, our results indicate that only $60 \%$ of patients received treatment within 3 months of diagnosis, which is consistent with previous studies ranging between $50 \%-70 \%{ }^{8,9}$ Early treatment and cytogenic response is linked to improved overall survival. ${ }^{23,24}$ While guidelines indicate that early treatment and monitoring is prudent, there can be multiple reasons for delayed first-line TKI treatment initiation, including lags in cytogenetic testing, other procedures, comorbidities, prior treatment with hydroxyurea for leukocytosis, access to care, or patient treatment decision. ${ }^{7,25}$

The FFS patients included in this study had additional inpatient and outpatient claims data, which allowed the ability to assess if delays in TKI initiation were because patients first received other systemic therapies (e.g., cyclophosphamide, cytarabine, and interferon alfa) or oral medications (e.g., cyclophosphamide, melphalan, and busulfan). Very few $(n=12)$ included FFS patients who received other therapies prior to TKI initiation (data not shown). Therefore, in this study, TKI treatment delays do not appear to be due to receipt of other infusion therapies.

Although there has been substantive adoption of TKIs, disparities by patient factors were observed. Patients aged 75 years and older were significantly less likely to receive a TKI than patients aged 65-74 years, which is consistent with past findings. ${ }^{8}$ Previous clinical trials have found that the molecular response and survival for patients treated with imatinib was similar for older patients when compared with younger patients. ${ }^{26,27}$

While older patients may benefit from TKIs, clinicians must consider the patient's overall health status. The prevalence of cardiovascular and other significant comorbidities has been cited as a factor in treatment selection. ${ }^{14,28}$ 


\section{FIGURE 2 Average Monthly Medicare Payments (TKI-Associated Health Care Cost) and Patient OOP Cost Among CML Patients Aged $\geq 65$ Years at Diagnosis Who Received TKI Therapy Within 1 Year of Diagnosis by Treatment Claim Year, 2077-2015 SEER-Medicare}

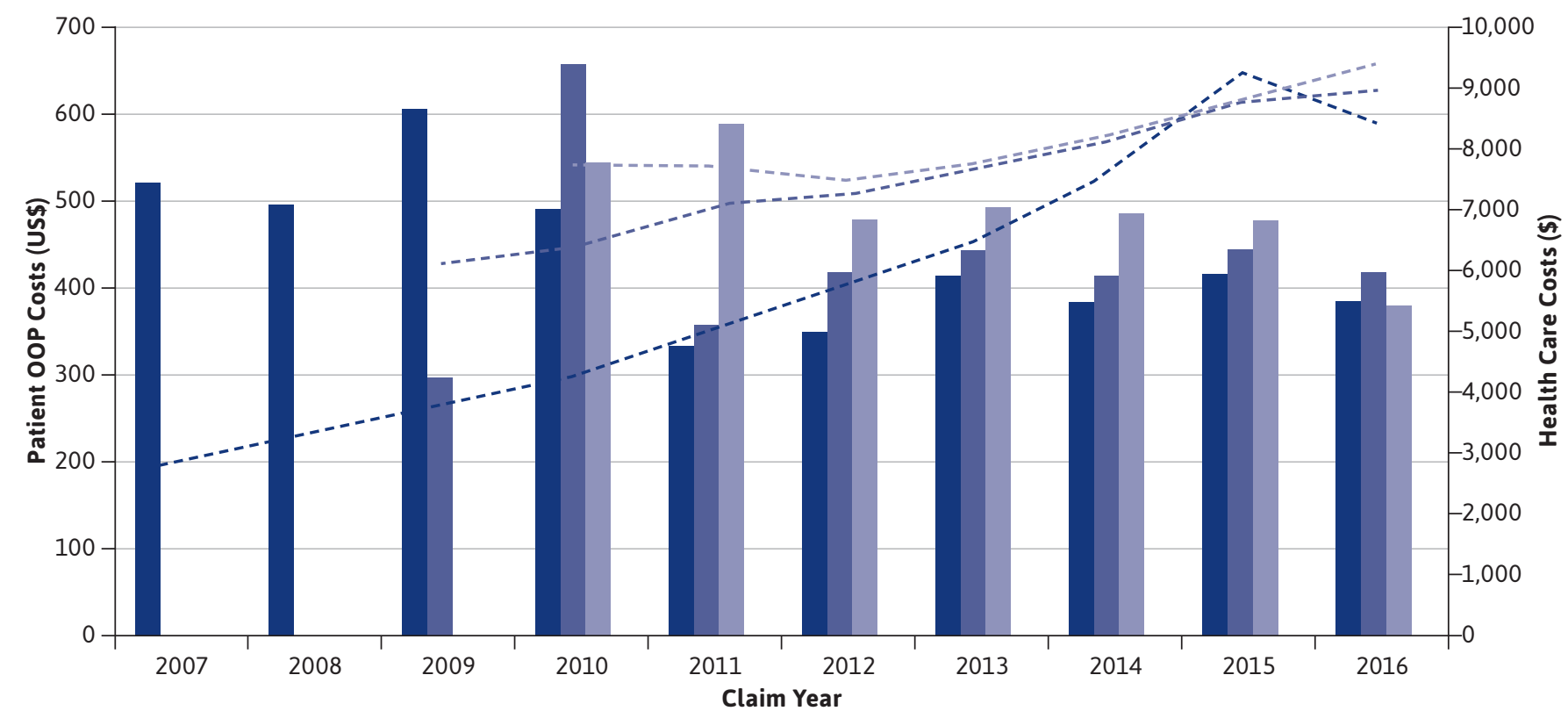

OOP Cost: $\square$ Imatinib

- ----- Imatinib

Dasatinib

--n-- Dasatinib

Nilotinib

- - - - - Nilotinib

$\mathrm{CML}=$ chronic myelogenous leukemia; $\mathrm{OOP}=$ out of pocket; $\mathrm{SEER}=$ Surveillance, Epidemiology, and End Results; TKI=tyrosine kinase inhibitor

Interestingly, age was still statistically significantly associated with slower initiation after adjustment for previous health status (CMS-HCC). Another possible explanation for lower TKI use in the very elderly is that some patients may have quality-of-life concerns related to taking TKIs, such as long-term adherence to medications, toxicities that affect activities of daily living, cognitive impairment, or financial toxicity, which may have influenced their decisions. ${ }^{29}$ Therefore, the reason that fewer elderly patients received TKIs is likely multifactorial, and further review of the factors that influence the clinical decision-making process is warranted. ${ }^{30-32}$

In agreement with previous studies, we also found that CML patients who were LIS ineligible under Part D were significantly less likely to have early initiation of a TKI than LIS-eligible patients. ${ }^{8}$ We found that this variation persisted regardless of HMO or FFS status. Patients who are LIS ineligible are responsible for paying premiums, deductibles, copayments, and coinsurance, which vary based on the plan and tier level of the drug. Fully LIS-eligible beneficiaries can have copayments reduced to a small fee (typically $\$ 3.30-$ $\$ 8.25$ per prescription fill), limiting OOP costs. ${ }^{33}$

The evaluation of cost at the health care and patient level was integral to enhancing interpretation of the association between receipt of TKI and LIS eligibility. The annual price estimates of the 3 TKIs assessed in the current study were near or greater than $\$ 100,000$, consistent with market cost estimates. ${ }^{34}$ The overall Medicare payments increased annually at a much higher rate than inflation, which reinforces concern for the affordability of these medications over time. ${ }^{35}$

While there was minimal variation in Medicare payments by LIS status, there were vast differences in OOP costs by LIS status. LIS-eligible patients receiving any TKI had an average monthly OOP cost of $<\$ 40$ (median $\$ 1$ ), whereas LIS-ineligible patients had monthly OOP costs responsibility averaging hundreds of dollars. In combination, the results of the regression model may suggest significantly increased TKI use among LIS-eligible patients because it is more affordable for subsidized patients to obtain 


\section{FIGURE 3 Average Monthly OOP Costs for TKIs Among CML Patients Aged $\geq 65$ Years at Diagnosis Who Received TKI Therapy Within 1 Year of Diagnosis by Part D LIS Status and Treatment Claim Year, 2007-2015 SEER-Medicare}

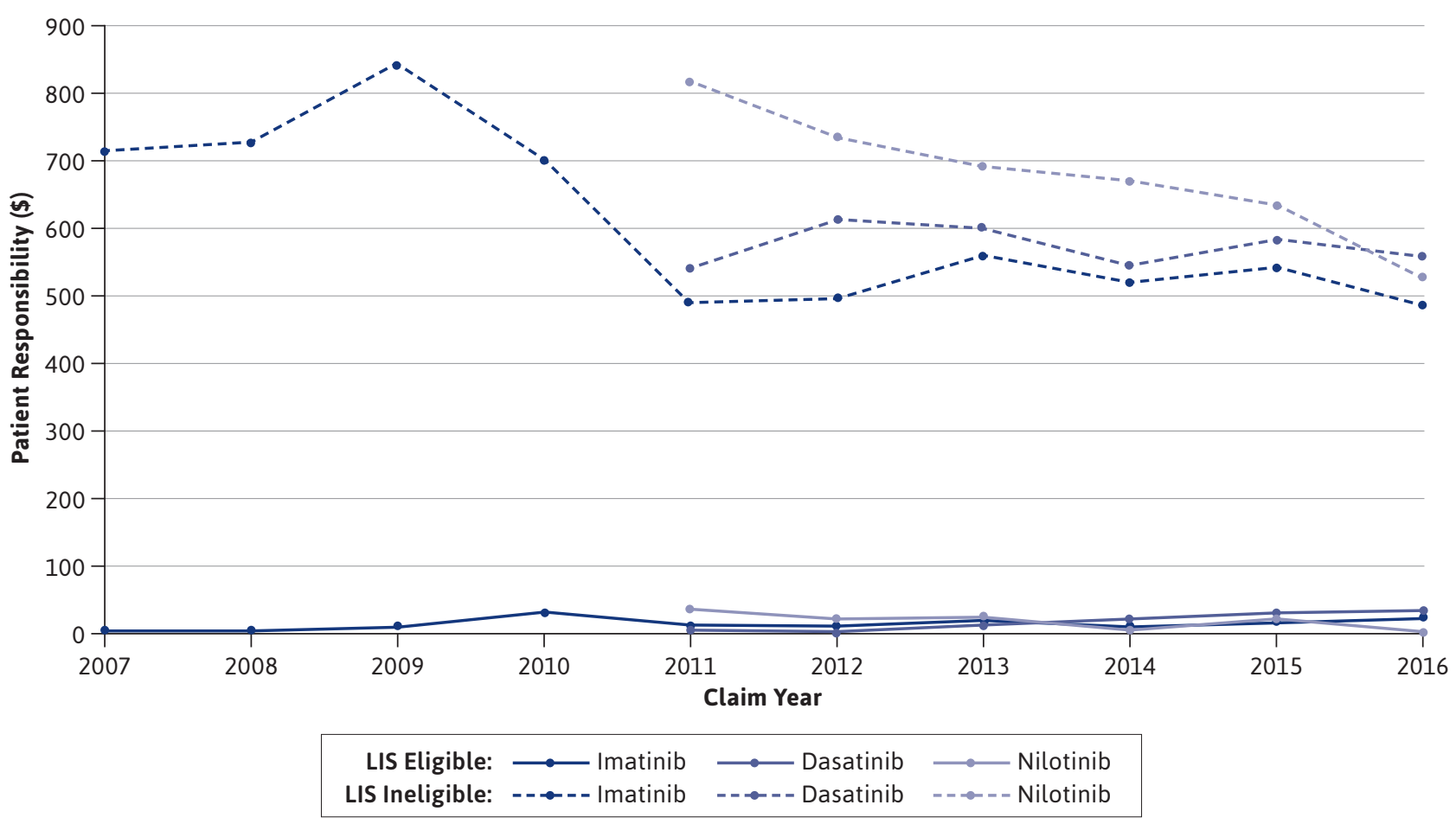

$\mathrm{CML}=$ chronic myelogenous leukemia; LIS = low-income subsidy; OOP=out of pocket; SEER: Surveillance, Epidemiology, and End Results; TKI=tyrosine kinase inhibitor.

their medications. ${ }^{36}$ High patient expenses have previously been shown to negatively affect treatment initiation and

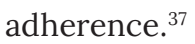

The cost of TKIs will likely continue to be a challenge for CML patients and the health care system. Our data show that imatinib, an effective drug with a well-studied cytogenic response and side-effect profile, continues to be the mainstay of CML treatment. Imatinib became available in generic form in early 2016, and the current study indicated an initial increase, leveling off shortly after market entry and resulting in mild OOP cost reductions that were noticeable for LIS-ineligible patients only, given that costs among LIS-eligible patients were fairly consistently negligible with mild fluctuations. Delays in treatment initiation related to LIS eligibility have been shown previously ${ }^{8,10}$; given that cost differences were still notable and persisted after the introduction of generic imatinib, economic barriers for elderly CML patients should continue to be evaluated.
Average monthly health care costs based on Medicare payments for imatinib was reduced by a few thousand dollars after the introduction of generic imatinib. While the introduction of generic imatinib may reduce the TKI health care costs by reducing individual drug payments, total TKI health care costs may be offset by increasing use of more costly second-generation TKIs. ${ }^{9}$

This study was able to examine the early impact of imatinib generic availability, although the overall market effect is likely not fully immediate for the consumer. ${ }^{38}$ Lower costs for patients are not only associated with treatment initiation, but can also be linked to improved adherence and, thus, improved survival.9,10,39 Furthermore, the use of dasatinib and nilotinib increased markedly from 2010 to 2016, reflecting the uptake of newer second-generation agents. If physicians opt to treat patients with dasatinib or nilotinib instead of generic imatinib as first-line therapy, there may not be cost savings for CML patients. 
Total cost to the health care system (e.g., payments, visits, and adverse drugs events) is complex, requiring additional data for complete evaluation. In addition, 3 new drugs were approved by the FDA in 2012, bosutinib, ponatinib, and the injectable protein synthesis inhibitor omacetaxine mepesuccinate (Synribo, used in drug resistant $\mathrm{CML}$ ), which are associated with even higher costs. Even if imatinib remains the first agent of choice, use of these other costly agents are likely to be clinically necessary, given primary and secondary imatinib resistance. Likewise, in this study nearly one third of patients received a second-line therapy (data not shown), consistent with another recently published study. ${ }^{40}$

The strengths of this study include being population-based, evaluating the introduction of imatinib generic with the most current longitudinal Part D claims data, inclusion of FFS and HMO populations, use of the CMS-HCC risk score as a proxy for previous health status, and ability to examine the effect of LIS eligibility on health care and patient OOP costs.

\section{LIMITATIONS}

This study has several limitations that are important to understand when interpreting the results. TKI utilization is only reflected through 2016 (lag time in SEER-Medicare linkage), limiting follow-up evaluation of the newest agents in patient care. Furthermore, claims were used to determine if a patient received a TKI. However, it was indeterminable if the patient was actively taking the agent or ingested the full dose. Given the expensive nature of these medications, it would be reasonable to believe that patients with claims for these therapies did indeed take them.

The assessment of first-line use relies on having a Medicare Part D claim for one of these agents after diagnosis, which reduced the study population to elderly persons with Part D coverage. Receipt of TKIs may have been underascertained because there was no information available on receipt of medication through other mechanisms, such as manufacturer samples or coupon rebate programs. ${ }^{41}$

Detailed information on CML phase, cytogenetic response, and genetic mutations was also not available. CML phase could affect the treatment pathway or receipt of other clinically appropriate therapies; cytogenetic response is a direct factor in clinical decision making. Clinicians may select certain TKIs based on cytogenetic testing for patients with primary mutations or requiring refractory treatment. If patients have accelerated or blast phase CML, they can be treated through induction chemotherapy, clinical trials, or transplant. Such factors may have affected treatment selection decisions to use more expensive agents, especially if genetic mutations confer primary resistance to imatinib. ${ }^{42}$

\section{Conclusions}

The introduction of TKIs has changed the disease course of CML, and providers have clearly prescribed these agents as first-line therapy for most of their patients, consistent with previous studies. Since 2010, there has been a shift in first-line therapy for CML, with decreased use of imatinib and increased use of dasatinib and nilotinib. The development of multiple firstline agents allows providers to select the therapeutic modality that is most appropriate for each patient. Newer agents offer the potential to overcome resistant mutations and varying side effects; however, this comes with escalating costs.

With additional years of data, this study indicated the sustained role of LIS eligibility on patient economic responsibility, even after the early introduction of generic imatinib. The substantial costs of these essential agents raise significant health care delivery questions around the sustained affordability of these long-term therapies in the elderly population, particularly among unsubsidized patients, and prompt important considerations around the use of generic drugs in clinical practice.

\section{DISCLOSURES}

No outside funding supported this study There are no reported conflicts of interest.

\section{ACKNOWLEDGMENTS}

The authors acknowledge Joan L. Warren $\mathrm{PhD}$, for her knowledge in establishing the study concept and for her scientific mentorship in the development of this manuscript.

\section{REFERENCES}

1. Howlader NA, Krapcho M, Miller D, et al eds. SEER Cancer Statistics Review, 19752013. National Cancer Institute. April 2016 Accessed October 28, 2020. https://seer. cancer.gov/archive/csr/1975 2013/

2. Siegel RL, Miller KD, Jemal A. Cancer statistics, 2018. CA Cancer J Clin. 2018;68(1):7-30.

3. Kantarjian H, O'Brien S, Jabbour E, et al. Improved survival in chronic myeloid leukemia since the introduction of imatinib therapy: a single-institution historical experience. Blood. 2012;119(9):1981-87.

4. Druker B, Guilhot F, O'Brien S, et al. Five-year follow-up of patients receiving imatinib for chronic myeloid leukemia. N Engl J Med. 2006;355(23):2408-17.

5. GLEEVEC (imatinib mesylate) tablets for oral use. Novartis Pharma. 2001. Accessed October 28, 2020. https://www. accessdata.fda.gov/drugsatfda_docs/ label/2008/021588s024lbl.pdf 
6. Jabbour E, Parikh SA, Kantarjian H, Cortes J. Chronic myeloid leukemia: mechanisms of resistance and treatment. Hematol Oncol Clin North Am. 2011;25(5):981-95.

7. Radich JP, Deininger M, Abboud CN, et al. Chronic myeloid leukemia, version 1.2019. NCCN Clinical Practice Guidelines in Oncology. J Natl Compr Canc Netw. 2018;16(9):1108-35. doi: 10.6004/ jnccn.2018.0071

8. Winn AN, Keating NL, Dusetzina SB. Factors associated with tyrosine kinase inhibitor initiation and adherence among medicare beneficiaries with chronic myeloid leukemia. J Clin Oncol. 2016;34(36):4323-28.

9. Kenzik KM, Bhatia R, Williams GR, Bhatia S. Medicare and patient spending among beneficiaries diagnosed with chronic myelogenous leukemia. Cancer. 2019;125(15):2570-78.

10. Shen C, Zhao B, Liu L, Shih YT. Financial burden for patients with chronic myeloid leukemia enrolled in Medicare Part D taking targeted oral anticancer medications. J Oncol Pract. 2017;13(2):e152-e162.

11. Lin PJ, Winn AN, Parsons SK, Neumann PJ, Weiss ES, Cohen JT. Linking costs and survival in the treatment of older adults with chronic myeloid leukemia: an analysis of SEER-Medicare data from 1995 to 2007. Med Care. 2016;54(4):380-85.

12. Warren JL, Klabunde CN, Schrag D, Bach PB, Riley GF. Overview of the SEERMedicare data: content, research applications, and generalizability to the United States elderly population. Med Care. 2002;40(8 Suppl):Iv-3-18.

13. Ortego A, Gaieski DF, Fuchs BD, et al. Hospital-based acute care use in survivors of septic shock. Crit Care Med. 2015;43(4):729-37.

14. Goldberg SL, Cortes JE, GambacortiPasserini C, et al. First-line treatment selection and early monitoring patterns in chronic phase-chronic myeloid leukemia in routine clinical practice: SIMPLICITY. Am J Hematol. 2017;92(11):1214-23.
15. Pope G, Kautter J, Ingber MJ, Freeman S, Sekar R, Newhart C. Evaluation of the CMS-HCC risk adjustment model. Final report. Centers for Medicare \& Medicaid Services. March 2011. Accessed October 28, 2020. https://www.cms.gov/ Medicare/Health-Plans/MedicareAdvtg SpecRateStats/downloads/Evaluation Risk_Adj_Model_2011.pdf

16. Li P, Kim MM, Doshi JA. Comparison of the performance of the CMS Hierarchical Condition Category (CMS-HCC) risk adjuster with the Charlson and Elixhauser comorbidity measures in predicting mortality. BMC Health Serv Res. 2010;10:245.

17. Efficace F, Cannella L. The value of quality of life assessment in chronic myeloid leukemia patients receiving tyrosine kinase inhibitors. Hematology Am Soc Hematol Educ Program. 2016;2016(1):170-79.

18. Tang L, Zhang H, Peng Y-Z, et al. Comparative efficacy and tolerability of front-line treatments for newly diagnosed chronic-phase chronic myeloid leukemia: an update network meta-analysis. BMC Cancer. 2019;19(1):849-49.

19. Eek D, Krohe M, Mazar I, et al. Patientreported preferences for oral versus intravenous administration for the treatment of cancer: a review of the literature. Patient Prefer Adherence. 2016;10:1609-21.

20. Hochhaus A, Larson RA, Guilhot F, et al. Long-term outcomes of imatinib treatment for chronic myeloid leukemia. New Engl J Med. 2017;376(10):917-27.

21. O'Brien SG, Guilhot F, Larson RA, et al. Imatinib compared with interferon and low-dose cytarabine for newly diagnosed chronic-phase chronic myeloid leukemia. N Engl J Med. 2003(348):994-1004.

22. Baccarani M, Efficace F, Rosti G. Moving towards patient-centered decision-making in chronic myeloid leukemia: assessment of quality of life and symptom burden. Haematologica. 2014;99(2):205-08.

23. Hanfstein B, Muller MC, Hehlmann R, et al. Early molecular and cytogenetic response is predictive for long-term progression-free and overall survival in chronic myeloid leukemia (CML). Leukemia. 2012;26(9):2096-102.
24. Quintas-Cardama A, Kantarjian H, Jones D, et al. Delayed achievement of cytogenetic and molecular response is associated with increased risk of progression among patients with chronic myeloid leukemia in early chronic phase receiving high-dose or standard-dose imatinib therapy. Blood. 2009;113(25):6315-21.

25. Bolle S, Smets EMA, Hamaker ME, Loos EF, van Weert JCM. Medical decision making for older patients during multidisciplinary oncology team meetings.

J Geriatr Oncol. 2019;10(1):74-83.

26. Rousselot P, Cony-Makhoul P, Nicolini F, et al. Long-term safety and efficacy of imatinib mesylate in elderly patients with chronic phase chronic myelogenous leukemia: results of the AFR04 study. Am J Hematol. 2013;88(1):1-4.

27. Proetel U, Pletsch N, Lauseker M, et al. Older patients with chronic myeloid leukemia ( $\geq 65$ years) profit more from higher imatinib doses than younger patients: a subanalysis of the randomized CML-Study IV. Ann Hematol. 2014;93(7):1167-76.

28. Jabbour E, Makenbaeva D, LingohrSmith M, Lin J. Use of real-world claim databases to assess prevalence of comorbid conditions relevant to the treatment of chronic myelogenous leukemia based on National Comprehensive Network treatment guidelines. Clin Lymphoma Myeloma Leuk. 2015;15(12):797-802.

29. Flynn KE, Atallah E. Quality of life and long term therapy in patients with chronic myeloid leukemia. Curr Hematol Malig Rep. 2016;11(2):80-85.

30. Wiggins CL, Harlan LC, Nelson HE, et al. Age disparity in the dissemination of imatinib for treating chronic myeloid leukemia. Am J Med. 2010;123(8):764.e1-9.

31. Alagiakrishnan K, Mah D, Padwal R. Classic challenges and emerging approaches to medication therapy in older adults. Discov Med. 2018;26(143):137-46.

32. To THM, Soo WK, Lane H, et al. Utilisation of geriatric assessment in oncology - a survey of Australian medical oncologists. J Geriatr Oncol. 2019;10(2):216-21. 
33. Medicare Payment Advisory Commission. Chapter 14: Status report on the Medicare prescription drug program (Part D). In: Report to the Congress: Medicare payment policy. March 2017. Accessed October 29, 2020. http:// www.medpac.gov/docs/default-source/ reports/mar17 medpac ch14.pdf2017

34. Experts in Chronic Myeloid Leukemia. The price of drugs for chronic myeloid leukemia (CML) Is a reflection of the unsustainable prices of cancer drugs: from the perspective of a large group of CML experts. Blood. 2013;121(22):4439-42.

35. Cubanski J, Neuman T. Assessing drug price increases in Medicare Part D and the implications of inflation limits. Kaiser Family Foundation. October 18, 2019. Accessed October 29, 2020. https://www. kff.org/medicare/issue-brief/assessingdrug-price-increases-in-medicare-partd-and-the-implications-of-inflationlimits/
36. U.S. Government Accountability Office. Medicare Part D: Spending BCS, and Cost-Containment Efforts for High-Cost Drugs Eligible for a Specialty Tier. 2010. Report to the Chairman, Subcommittee on Health, Committee on Ways and Means, House of Representatives. GAO-10-242. January 2020. Accessed October 29, 2020. https:// www.gao.gov/new.items/d10242.pdf

37. Dusetzina SB, Winn AN, Abel GA, Huskamp HA, Keating NL. Cost sharing and adherence to tyrosine kinase inhibitors for patients with chronic myeloid leukemia. J Clin Oncol. 2014;32(4):306-11.

38. Chen CT, Kesselheim AS. Journey of generic imatinib: a case study in oncology drug pricing. J Oncol Pract. 2017;13 (6):352-55.
39. Cole AL, Jazowski SA, Dusetzina SB. Initiation of generic imatinib may improve medication adherence for patients with chronic myeloid leukemia. Pharmacoepidemiol Drug Saf. 2019;28(11):1529-33.

40. Banegas MP, Rivera DR, O'KeeffeRosetti MC, et al. Long-term patterns of oral anticancer agent adoption, duration, and switching in patients with CML. J Natl Compr Canc Netw. 2019;17(10):1166-72.

41. Sloan C, Zafar SY. Ask early and ask often: how discussing costs could save your patient's life. N C Med J. 2018;79(1):39-42.

42. Pfeifer H, Lange T, Wystub S, et al. Prevalence and dynamics of BCR-ABL kinase domain mutations during imatinib treatment differ in patients with newly diagnosed and recurrent BCR-ABL positive acute lymphoblastic leukemia. Leukemia. 2012;26(7):1475-81. 